\title{
Composição de bandos mistos de aves no Parque Estadual das Araucárias, oeste de Santa Catarina, Brasil
}

\author{
Ivo Rohling Ghizoni-Jr \\ Caipora Cooperativa para Conservação da Natureza \\ Av. Desembargador Vitor Lima, 260/513, CEP 88.040-400, Florianópolis - SC, Brasil \\ ivoghizoni@yahoo.com.br
}

Submetido em 12/11/2008

Aceito para publicação em 18/03/2009

\section{Resumo}

Bandos mistos de aves são associações de duas ou mais espécies possivelmente formados pela maximização de forrageio e/ou minimização de predação. Diversos estudos com bandos mistos vêm sendo realizados no sul do Brasil, mas nenhum em Floresta Ombrófila Mista. Em função disso, foi realizado um acompanhamento de bandos mistos florestais no Parque Estadual das Araucárias (PEA) em São Domingos, oeste de Santa Catarina, de 2006 a 2007. Foram registradas 56 espécies de aves nos 29 bandos encontrados, das quais 36 espécies (64\%) apresentaram frequência de ocorrência superior a 10\%. Os bandos foram formados, em média por 11 espécies e as mais abundantes foram Parula pitiayumi (72\%), Hemithraupis guira (65\%), Basileuterus culicivorus (62\%), Lepidocolaptes falcinellus (62\%), Veniliornis spilogaster (55\%) e Sittasomus griseicapillus (51\%). Aparentemente não há uma espécie nuclear que atue como responsável pela formação e coesão dos bandos mistos, mas um complexo de espécies nucleares que favorecem que os bandos sejam relativamente grandes.

Unitermos: aves, bandos mistos, Floresta Ombrófila Mista, Santa Catarina

\section{Abstract}

Composition of mixed-species flocks of forest birds in the Parque Estadual das Araucárias, western Santa Catarina State, Brazil. Mixed-species flocks are associations of two or more species possibly derived from the maximization of foraging and/or minimization of predation. Several studies with mixed-species flocks have been carried out in southern Brazil, but none of them in a native Araucaria forest. Mixed-species bird flocks were surveyed between 2006 to 2007 at the Parque Estadual das Araucárias (PEA), in São Domingos, in the western part of the state of Santa Catarina. Fifty-six species were recorded in 29 flocks and the average number of birds per flock was 11 . Of these species, 36 (64\%) had a frequency of occurrence that was higher than $10 \%$. The most abundant species were Parula pitiayumi (72\%), Hemithraupis guira (65\%), Basileuterus culicivorus (62\%), Lepidocolaptes falcinellus (62\%), Veniliornis spilogaster (55\%) and Sittasomus griseicapillus (51\%). Apparently, there was no nuclear species that assumed responsibility for the mixed flock's constitution and cohesion, but instead a complex of nuclear species that acted in way that favored the relatively large sizes of the flocks.

Key words: birds, mixed-species flocks, Native Araucaria forest, Santa Catarina 


\section{Introdução}

Bandos mistos de aves são aglomerações de duas ou mais espécies que interagem durante certo tempo, espaço e direção (Moynihan, 1962). Duas hipóteses não excludentes são levantadas para a explicação adaptativa de tal comportamento: a maximização de forrageio (Wilson, 1975) e a minimização da predação (Moynihan, 1962; Morse, 1977; Powell, 1979). Tais aglomerações podem interferir no comportamento alimentar (Develey e Peres, 2000) ou mesmo na altura de forrageio (Machado e Rodrigues, 2000) de algumas espécies. Bandos mistos de aves florestais formam-se a partir de espécies "nucleares" (Moynihan, 1962) que normalmente têm altos índices de associação, coloração críptica e emitem chamados constantes, sendo responsáveis pela formação, direção e coesão dos bandos mistos. Estas espécies podem também servir de sentinela na aproximação de um predador (Munn, 1986). A riqueza de espécies e frequência de associação de bandos mistos são influenciados pelas estações do ano, disponibilidade de alimento, estação reprodutiva (Develey e Peres, 2000), altitude (Terborgh, 1977) e fragmentação florestal (Maldonado-Coelho e Marini, 2003).

Vários estudos têm sido realizados para entender a dinâmica da composição e estrutura dos bandos mistos de aves no Brasil, sendo a maioria em Floresta Atlântica do sudeste (e.g. Davis, 1946; Aleixo, 1997; Machado, 1991; 1997; 1999; 2002; Develey e Peres, 2000; Machado e Rodrigues, 2000; Develey, 2001; Maldonado-Coelho e Marini, 2000; 2003; 2004). Estudos de bandos mistos no sul do Brasil incluem os de Moraes e Krul (1995); Marterer (1996); Ghizoni-Jr. e Azevedo (2006) e Brandt (2008) em Santa Catarina, e Silveira (2006) no Rio Grande do Sul. Estes estudos, no entanto, limitam-se à vertente atlântica, e até hoje nenhum trabalho com bandos mistos foi realizado na Floresta Ombrófila Mista, cuja composição de espécies e estacionalidade é bem diferente.

Este estudo tem por objetivo descrever a estrutura e composição dos bandos mistos de aves no Parque Estadual das Araucárias (PEA), oeste catarinense, em Floresta Ombrófila Mista.

\section{Material e Métodos}

O PEA é um fragmento florestal com cerca de 700ha, margeado por áreas de agricultura e pecuária. Está situado nos municípios de São Domingos e Galvão, oeste de Santa Catarina (26 $27^{\circ}$ 'S e $52^{\circ} 34^{\prime}$ W; Figura 1), a uma altitude média de $650 \mathrm{~m}$. O clima local é do tipo Cfa de Köeppen, mesotérmico úmido, com verões quentes e sem estação seca definida, com chuvas distribuídas ao longo de todo o ano $(1400 \mathrm{~mm})$ e temperatura média anual de $18,5^{\circ} \mathrm{C}$ (GAPLAN, 1986). A vegetação constituinte do PEA pertence à Floresta Ombrófila Mista (FOM), cujo dossel é formado predominantemente por Araucaria angustifolia. A porção mais elevada do PEA sofreu corte seletivo de indivíduos de A. angustifolia, sendo por isso denominada "mata branca". As visitas foram trimestrais, entre abril de 2006 a setembro de 2007. A parte do PEA onde foram realizados os levantamentos dos bandos está localizada no município de São Domingos, em uma região onde há maior aglomeração de araucárias, em torno de $600 \mathrm{~m}$ de altitude.

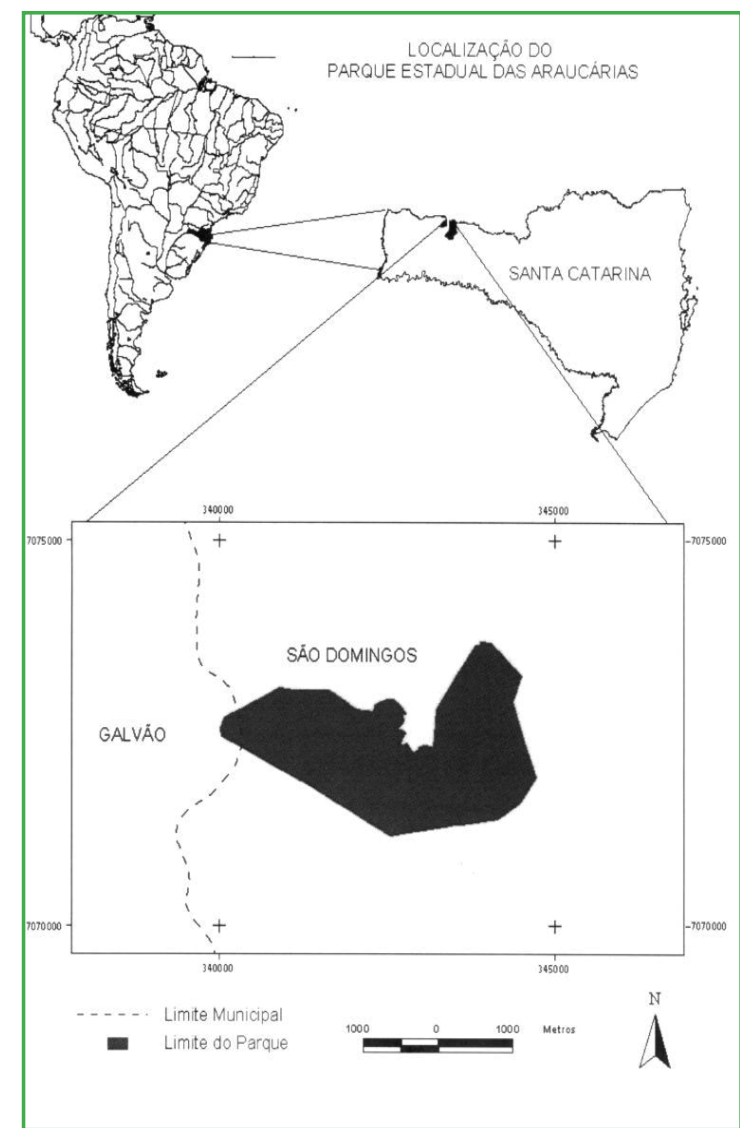

FIGURA 1: Localização do Parque Estadual das Araucárias (PEA) nos municípios de São Domingos e Galvão, oeste do estado de Santa Catarina. 
Trilhas e estradas foram percorridas dentro do PEA, numa velocidade de $2-3 \mathrm{~km} / \mathrm{h}$, totalizando cerca de $60 \mathrm{~h}$ de esforço amostral. Para certificação de que formavam realmente um bando misto, estes foram seguidos por pelo menos cinco minutos para verificação da direção, coesão e interação entre os indivíduos (Herrera, 1979). Atendidas estas condições, as espécies que compunham o bando foram identificadas com auxílio de um binóculo 10x42. A nomenclatura taxonômica utilizada seguiu o CBRO (2008).

A frequência de ocorrência (FO) de cada espécie foi estimada através da razão entre o número de vezes que uma espécie foi registrada participando de um bando misto e o número total de bandos mistos observados. Para a categorização das espécies quanto à participação nos bandos, seguiu-se Maldonado-Coelho e Marini (2000), sendo consideradas espécies-nucleares aquelas com $\mathrm{FO} \geq 50 \%$ dos bandos mistos. As demais espécies foram categorizadas como espécies regulares (FO entre 10 e $49 \%$ dos bandos) ou espécies ocasionais (FO < $10 \%$ dos bandos). Todos os cálculos foram realizados no programa BioEstat 2.0 (Ayres et al., 2000). Os valores foram apresentados na forma de média \pm desviopadrão.

\section{Resultados}

Foram observados 29 bandos mistos e registradas 56 espécies de aves participantes. O número médio de espécies por bando foi 11,1 \pm 4,92 (mínimo de 4 máximo de 21 espécies, $\mathrm{n}=322$ indivíduos). Trinta e seis espécies (64\%) foram consideradas regulares ou nucleares, estando presentes em mais de $10 \%$ do total de bandos amostrados (Tabela 1). As demais $(n=20$; $36 \%$ ), tiveram baixas associações e foram consideradas ocasionais (Figura 2).

As espécies que apresentaram maiores frequências de ocorrência foram Parula pitiayumi (72\%), Hemithraupis guira (65\%), Basileuterus culicivorus (62\%), Lepidocolaptes falcinellus (62\%), Veniliornis spilogaster (55\%), Sittasomus griseicapillus (51\%), Colaptes melanochloros (44\%), Pachyramphus castaneus (44\%) e Trichothraupis melanops (41\%).

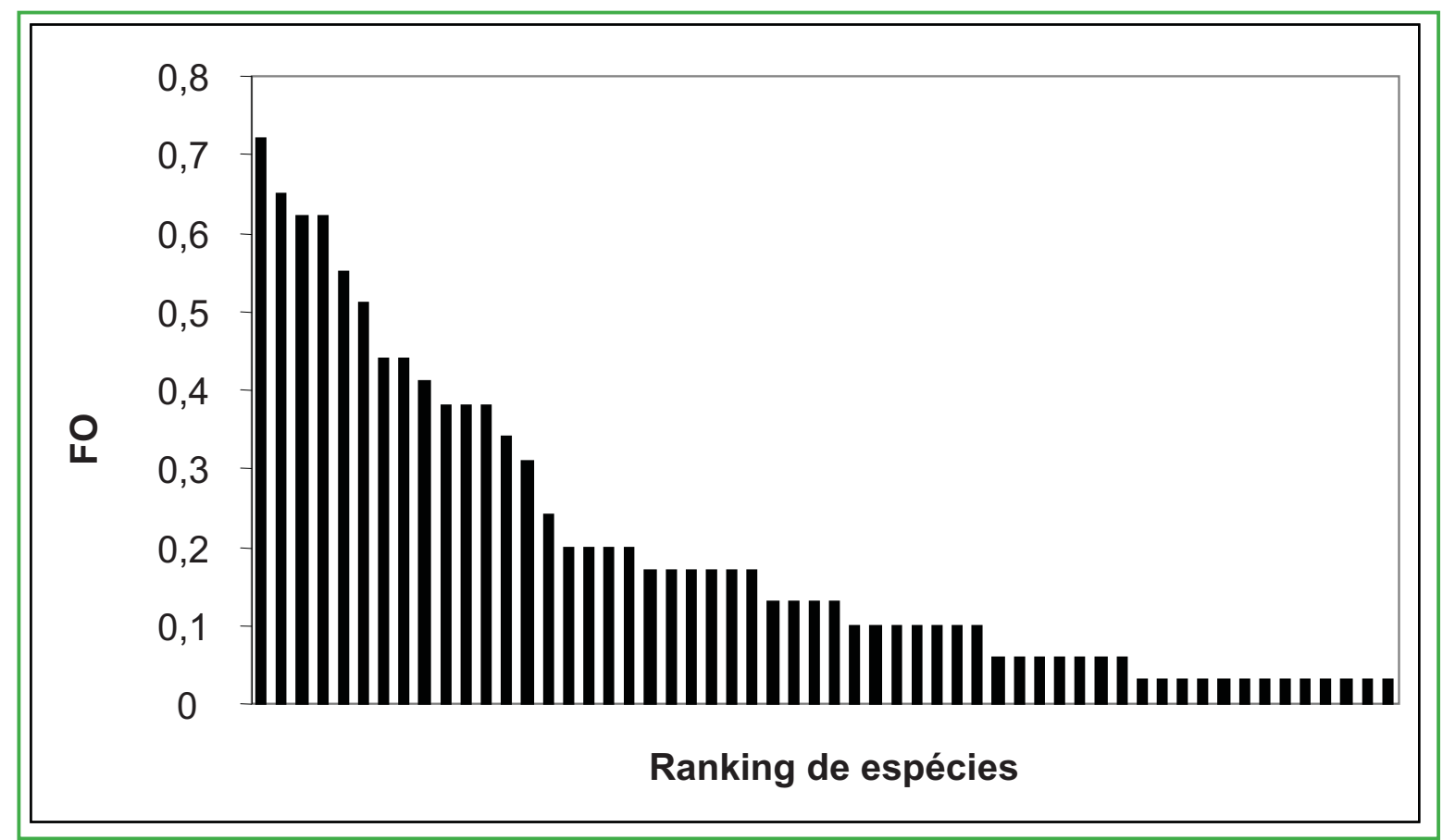

FIGURA 2: Frequência de ocorrência (FO) das 56 espécies de aves registradas nos bandos mistos no Parque Estadual das Araucárias, Santa Catarina, sul do Brasil. 
TABELA 1: Frequência de ocorrência (FO) e número de registros (entre parênteses) das espécies de aves de bandos mistos florestais no Parque Estadual das Araucárias, em Floresta Ombrófila Mista, oeste de Santa Catarina. Status: N = nuclear; R = regular; $\mathrm{O}=$ ocasional.

\begin{tabular}{|c|c|c|}
\hline Espécies & Status & FO \\
\hline 1. Parula pitiayumi & $\mathrm{N}$ & $0,72(21)$ \\
\hline 2. Hemithraupis guira & $\mathrm{N}$ & 0,65 (19) \\
\hline 3. Basileuterus culicivorus & $\mathrm{N}$ & $0,62(18)$ \\
\hline 4. Lepidocolaptes falcinellus & $\mathrm{N}$ & $0,62(18)$ \\
\hline 5. Veniliornis spilogaster & $\mathrm{N}$ & $0,55(16)$ \\
\hline 6. Sittasomus griseicapillus & $\mathrm{N}$ & $0,51(15)$ \\
\hline 7. Colaptes melanochloros & $\mathrm{R}$ & $0,44(13)$ \\
\hline 8. Pachyramphus castaneus & $\mathrm{R}$ & $0,44(13)$ \\
\hline 9. Trichothraupis melanops & $\mathrm{R}$ & $0,41(12)$ \\
\hline 10. Trogon surrucura & $\mathrm{R}$ & $0,38(11)$ \\
\hline 11. Cyclarhis gujanensis & $\mathrm{R}$ & $0,38(11)$ \\
\hline 12. Dendrocolaptes platyrostris & $\mathrm{R}$ & $0,38(11)$ \\
\hline 13. Thamnophilus caerulescens & $\mathrm{R}$ & $0,34(10)$ \\
\hline 14. Conirostrum speciosum & $\mathrm{R}$ & $0,31(9)$ \\
\hline 15. Leptopogon amaurocephalus & $\mathrm{R}$ & $0,24(7)$ \\
\hline 16. Pachyramphus viridis & $\mathrm{R}$ & $0,20(6)$ \\
\hline 17. Cranioleuca obsoleta & $\mathrm{R}$ & $0,20(6)$ \\
\hline 18. Pyrrhocoma ruficeps & $\mathrm{R}$ & $0,20(6)$ \\
\hline 19. Pipraeidea melanonota & $\mathrm{R}$ & $0,20(6)$ \\
\hline 20. Basileuterus leucoblepharus & $\mathrm{R}$ & $0,17(5)$ \\
\hline 21. Poecilotriccus plumbeiceps & $\mathrm{R}$ & $0,17(5)$ \\
\hline 22. Xenops rutilans & $\mathrm{R}$ & $0,17(5)$ \\
\hline 23. Tolmomyias sulphurescens & $\mathrm{R}$ & $0,17(5)$ \\
\hline 24. Poospiza cabanisi & $\mathrm{R}$ & $0,17(5)$ \\
\hline 25. Camptostoma obsoletum & $\mathrm{R}$ & $0,17(5)$ \\
\hline 26. Turdus rufiventris & $\mathrm{R}$ & $0,13(4)$ \\
\hline 27. Tachyphonus coronatus & $\mathrm{R}$ & $0,13(4)$ \\
\hline 28. Saltator maxillosus & $\mathrm{R}$ & $0,13(4)$ \\
\hline 29. Phylloscartes ventralis & $\mathrm{R}$ & $0,13(4)$ \\
\hline 30. Contopus cinereus & $\mathrm{R}$ & $0,10(3)$ \\
\hline 31. Saltator similis & $\mathrm{R}$ & $0,10(3)$ \\
\hline 32. Cacicus chrysopterus & $\mathrm{R}$ & $0,10(3)$ \\
\hline 33. Cyanocorax chrysops & $\mathrm{R}$ & $0,10(3)$ \\
\hline 34. Syndactyla rufosuperciliata & $\mathrm{R}$ & $0,10(3)$ \\
\hline 35. Dysithamnus mentalis & $\mathrm{R}$ & $0,10(3)$ \\
\hline 36. Turdus amaurochalinus & $\mathrm{R}$ & $0,10(3)$ \\
\hline 37. Phyllomyias burmeisteri & $\mathrm{O}$ & $0,06(2)$ \\
\hline 38. Phyllomyias griseocapilla & $\mathrm{O}$ & $0,06(2)$ \\
\hline 39. Phyllomyias virescens & $\mathrm{O}$ & $0,06(2)$ \\
\hline 40. Zonotrichia capensis & $\mathrm{O}$ & $0,06(2)$ \\
\hline 41. Synallaxis spixi & $\mathrm{O}$ & $0,06(2)$ \\
\hline 42. Philydor rufum & $\mathrm{O}$ & $0,06(2)$ \\
\hline 43. Troglodytes musculus & $\mathrm{O}$ & $0,06(2)$ \\
\hline
\end{tabular}

\begin{tabular}{lll} 
44. Vireo olivaceus & $\mathrm{O}$ & $0,03(1)$ \\
45. Cyanoloxia brissonii & $\mathrm{O}$ & $0,03(1)$ \\
46. Colonia colonus & $\mathrm{O}$ & $0,03(1)$ \\
47. Piculus aurulentus & $\mathrm{O}$ & $0,03(1)$ \\
48. Sirystes sibilator & $\mathrm{O}$ & $0,03(1)$ \\
49. Capsiempis flaveola & $\mathrm{O}$ & $0,03(1)$ \\
50. Piaya cayana & $\mathrm{O}$ & $0,03(1)$ \\
51. Myiodynastes maculatus & $\mathrm{O}$ & $0,03(1)$ \\
52. Turdus albicollis & $\mathrm{O}$ & $0,03(1)$ \\
53. Turdus leucomelas & $\mathrm{O}$ & $0,03(1)$ \\
54. Sicalis flaveola & $\mathrm{O}$ & $0,03(1)$ \\
55. Cissopis leveriana & $\mathrm{O}$ & $0,03(1)$ \\
56. Synallaxis cinerascens & $\mathrm{O}$ & $0,03(1)$ \\
\hline
\end{tabular}

\section{Discussão}

O número médio de espécies participantes por bando misto no PEA $(11,1)$ é relativamente alto se comparado com outros trabalhos na vertente atlântica (e.g. Develey e Peres, 2000; Ghizoni-Jr e Azevedo, 2006; Silveira, 2006). Entretanto, números semelhantes foram encontrados por Stotz (1993) $(\sim 11,2)$ em Floresta Atlântica do Espírito Santo e Aleixo (1997) $(\sim 10,2) \mathrm{em}$ floresta semidecídua do interior de São Paulo. Bandos mistos são maiores na Amazônia, onde há maior número de espécies dependentes dos bandos e com isso há maior especialização desses integrantes dos bandos (Terborgh, 1977), diferente do que acontece no sul do Brasil.

NoPEAseis espécies destacaram-se por apresentarem alta frequência nos bandos (espécies nucleares) e $a$ priori parecem ter importância semelhante como espécies nucleares formando um 'complexo-núcleo', como observado por Machado (2002) em Floresta Atlântica de São Paulo. Além disso, tais espécies são responsáveis por arregimentar um maior número de espécies participantes dos bandos mistos e isto pode ser o motivo da alta riqueza de espécies e de associações encontradas no PEA se comparado com outros trabalhos realizados na vertente atlântica (e.g. Develey e Peres, 2000; GhizoniJr e Azevedo, 2006; Silveira, 2006; Brandt, 2008). Essa arregimentação deve-se a maior movimentação no ambiente onde os bandos passam, atraindo espécies durante certo período, mesmo que essas não sejam fiéis seguidoras dos bandos (Aleixo, 1997).

Dentre as espécies participantes dos bandos mistos do PEA, os Passeriformes correspondem a 90\%, 
assim como o encontrado por Silveira (2006) e Brandt (2008). Dentre as seis espécies mais frequentes, três são escaladoras de troncos (S. griseicapillus, L. falcinellus e $V$. spilogaster) e as outras são insetívoras e onívoras de copa (P. pitiayumi, H. guira e B. culicivorus). A maioria dessas espécies nucleares apresenta colorações crípticas e vocalizam constantemente. Tanto os dendrocolaptídeos como as outras espécies nucleares são consideradas integrantes comuns dos bandos mistos em outras regiões (Machado, 1999; Ghizoni-Jr. e Azevedo, 2006; Silveira, 2006).

A composição de espécies com maiores índices de associação no PEA é similar ao observado por Aleixo (1997) em floresta semidecídua de São Paulo. Não necessariamente as espécies eram as mesmas, mas sim seus congêneres (e.g., H. ruficapilla e H. guira, Xiphorhynchus fuscus e L. falcinellus) foram as mais frequentes. Essas diferenças na composição e frequência das espécies em bandos mistos em um determinado local ou região são em decorrência da composição da comunidade e como esses organismos se organizam na dinâmica dos bandos mistos (Ghizoni-Jr. e Azevedo, 2006; Silveira, 2006; Brandt, 2008).

A Floresta Ombrófila Mista cobria cerca de 30\% do planalto do sul do Brasil (Leite, 2002). Hoje é uma das tipologias mais ameaçadas do bioma Mata Atlântica e cobre apenas $0,7 \%$ da área original (MMA, 2002), distribuída geralmente em pequenos fragmentos esparsos. Apesar desse seu atual status de conservação, nenhum trabalho com bandos mistos havia sido realizado. Um maior esforço se faz necessário para se elucidar todas as características e relações dos bandos mistos com o ambiente e como elas podem estar interligadas (Develey, 2001).

\section{Agradecimentos}

À ETS e à CEC pela logística e a toda equipe de monitoramento da fauna de vertebrados terrestres da Usina Hidrelétrica Quebra Queixo (Paulo A. Hartmann, Luis O. M. Giasson, Jorge J. Cherem, Sérgio Althoff, Ayrton Schmidt, Célio Testoni e Karine Abati) e ao Clóvis Poli, Ênio Loesch e Juarez Camera pela ajuda em campo. À Ana Paula Silveira pelo abstract e a Jorge J.
Cherem, Claudia S. Brandt e os consultores pelas críticas e sugestões ao manuscrito.

\section{Referências}

Aleixo, A. 1997. Composition of mixed-species bird flocks and abundance of flocking species in a semideciduous forest of southeastern Brazil. Ararajuba, 5: 11-18.

Ayres, M.; Ayres Jr., M.; Ayres, D. L.; Santos, A. S. 2000. BioEstat 2.0. Sociedade Civil Mamirauá/MCT-CNPq, Brasília, Brasil, 259pp.

Brandt, C. S. 2008. Ecologia de bandos mistos de aves em uma paisagem de floresta atlântica no sul do Brasil. Dissertação de Mestrado. Universidade Federal do Rio Grande do Sul, Brasil, $53 \mathrm{pp}$.

CBRO - Comitê Brasileiro de Registros Ornitológicos. 2008. Listas das aves do Brasil. Versão 05/10/2008. Disponível em $<$ http://www.cbro.org.br>. Acesso em 20 de outubro de 2008. Davis, D. E. 1946. A seasonal analysis of mixed flocks of birds in Brazil. Ecology, 27 (2): 168-181.

Develey, P. F. 2001. Os bandos mistos nas florestas neotropicais. In: Albuquerque, J.; Cândido-Jr, J. F.; Straube, F. C. \& Roos, A. L. (Eds.). Ornitologia e conservação: Da ciência às estratégias. Unisul, Tubarão, Brasil, p.39-48.

Develey, P. F.; Peres, C. A. 2000. Resource seasonality and the structure of mixed species bird flocks in a coastal Atlantic forest of southeastern Brazil. Journal of Tropical Ecology, 16: 33-53.

GAPLAN, 1986. Atlas de Santa Catarina. Gabinete de Planejamento e Coordenação Geral, Rio de Janeiro, Brasil, 173pp.

Ghizoni-Jr, I. R.; Azevedo, M. A. G. 2006. Composição de bandos mistos florestais de sub-bosque em áreas de encosta e planície da Floresta Atlântica de Santa Catarina, sul do Brasil. Biotemas, 19 (2): 47-53.

Herrera, C. M. 1979. Ecological aspects of heteroespecific flocks formation in a Mediterranean passerine bird community. Oikos, 33: 85-89.

Leite, P. F. 2002. Contribuição ao conhecimento fitoecológico do sul do Brasil. Ciência \& Ambiente, 24: 51-73.

Machado, C. G. 1991. Estrutura, composição e dinâmica de bandos mistos de aves na Mata Atlântica do alto da Serra do Paranapiacaba, SP. Dissertação de Mestrado, Universidade Estadual de Campinas, Brasil, 79pp.

Machado, C. G. 1997. Vireo olivaceus (Vireonidae, Aves): Uma espécie migratória nos bandos mistos de aves da Mata Atlântica do sudeste brasileiro. Ararajuba, 1: 62-64.

Machado, C. G. 1999. A composição dos bandos mistos de aves na Mata Atlântica da serra de Paranapiacaba no sudeste brasileiro. Revista Brasileira de Biologia, 59 (1): 75-85.

Machado, C. G. 2002. As espécies-núcleo dos bandos mistos de aves da Mata Atlântica da Serra de Paranapiacaba, no sudeste brasileiro. Sitientibus, 2 (1/2): 85-90.

Machado, C. G.; Rodrigues, M. R. 2000. Alteração de altura de forrageamento de espécies de aves quando associadas a bandos mistos. In: Alves, M. A. S.; Silva, J. M. C.; Sluys, M. V.; Bergallo, 
H. G. \& Rocha, C. F. D. (Eds). A ornitologia no Brasil: Pesquisa atual e perspectivas. UERJ, Rio de Janeiro, Brasil, p.231-237.

Maldonado-Coelho, M.; Marini, M. Â. 2000. Effects of forest fragment size and successional stage of mixed-species bird flocks in southeastern Brazil. Condor, 102: 585-594.

Maldonado-Coelho, M.; Marini, M. Â. 2003. Composição de bandos mistos de aves em fragmentos de Mata Atlântica no sudeste do Brasil. Papéis Avulsos de Zoologia, 43 (3): 31-54.

Maldonado-Coelho, M.; Marini, M. Â. 2004. Mixed-species bird flocks from Brazilian Atlantic forest: the effects of forest fragmentation and seasonality on their size, richness and stability. Biological Conservation, 116: 19-26.

Marterer, B. T. P. 1996. Avifauna do Parque Botânico do Morro do Baú. Riqueza, aspectos de frequência e abundância. Fatma, Florianópolis, Brasil, 74pp.

MMA - Ministério do Meio Ambiente. 2002. Proposta do grupo de trabalho preservação e recuperação da Floresta Ombrófila Mista no Estado de Santa Catarina. Portaria Ministerial 49 de 06 de fevereiro de 2002, Brasília, Brasil, p.77.

Moraes, V. S.; Krul, R. 1995. Composição e finalidades de agrupamentos heteroespecíficos de aves em ambientes naturais do Paraná e Santa Catarina. Biotemas, 8 (2): 63-73.
Morse, D. H. 1977. Feeding behavior and predator avoidance in heterospecific groups. BioScience, 27: 332-339.

Moynihan, M. 1962. The organization and probable evolution of some mixed-species flocks of neotropical birds. Smithsonian Miscellaneous Collections, 143: 1-140.

Munn, C. A. 1986. Birds that "cry wolf”. Nature, 319: 143-145.

Powell, G. V. N. 1979. Structure and dynamics of interespecific flocks in a neotropical mid-elevation forest. The Auk, 96: 375390 .

Silveira, A. B. 2006. Composição, estrutura e sazonalidade de bandos mistos em um remanescente de floresta paludosa no sul do Brasil. Dissertação de Mestrado, Universidade Federal do Rio Grande do Sul, Brasil, 134pp.

Stotz, D. F. 1993. Geographic variation in species composition of mixed species flocks in lowland humid forest in Brazil. Papéis Avulsos de Zoologia, 38: 61-75.

Terborgh, J. 1977. Bird species diversity on an Andean elevation gradient. Ecology, 58: 1007-1019.

Wilson, E. O. 1975. Sociobiology: The new synthesis. Harvard University Press, Cambridge, USA, 697pp. 